\title{
Fine Temporal Properties of Center-Surround Interactions in Motion Revealed by Reverse Correlation
}

\author{
Duje Tadin, Joseph S. Lappin, and Randolph Blake \\ Vanderbilt Vision Research Center and Department of Psychology, Vanderbilt University, Nashville, Tennessee 37203
}

\begin{abstract}
Center-surround interactions are a key property of visual motion mechanisms. Using a temporal reverse correlation method with human observers, we investigated perceptual interactions between a brief center motion $(\sim 20 \mathrm{~ms})$ and a surround that moved up-down with a new direction chosen randomly every $5 \mathrm{~ms}$. The aim was to reveal interactions between center and surround motions and their dependency on relative direction, contrast, and timing. Hypothesizing that surround computation involves different neural circuitry than the center response, we manipulated surround contrast to affect the relative timing of center and surround signals. The reverse correlation analysis yielded temporal profiles of surround influence indicating, in $5 \mathrm{~ms}$ steps, the time course of the effect of the surround on the discriminability of center motion. The resulting temporal profiles varied systematically with contrast: as surround contrast decreased, both the latency and duration of its influence increased. This finding, consistent with longer and variable neural response latencies at low contrast, psychophysically reveals fine-scale temporal interactions between center and surround signals. Additionally, the strength of surround influence was correlated with psychophysical thresholds for discriminating center motion. The directionality of this relationship, however, depended only on center contrast. When center motion was high contrast, poor direction discrimination was associated with an increased probability of same-direction surround motions. Low-contrast center motion, however, was more discriminable when surrounded by motion in the same direction, regardless of surround contrast. This suggests that the previously reported adaptive nature of center-surround interactions in motion is driven primarily by the visibility of the center motion signals.
\end{abstract}

Key words: vision; visual motion; center-surround; contrast; reverse correlation; inhibition

\section{Introduction}

A significant portion of the neural analysis of visual input transpires within $150 \mathrm{~ms}$ after the stimulus onset (Thorpe et al., 1996). Within that time, different stimulus properties, such as color and motion, are processed and perceived at different times (Moutoussis and Zeki, 1997; Nishida and Johnston, 2002; Bedell et al., 2003). Delayed signals arrive via feedforward, feedback, and lateral connections, and together those signals play a fundamental role in neural processing of visual input (Lamme et al., 1998). Furthermore, stimulus properties such as contrast and velocity affect neuronal latencies (Nowak and Bullier, 1997), introducing stimulus-dependent delays. This hodgepodge of delays and asynchronies, although often obvious at the single-cell level, typically is hidden from phenomenal experience and is only revealed psychophysically by carefully designed experiments.

We psychophysically investigated temporal properties of center-surround interactions in motion. Center-surround processing is inaugurated in the retina, and it recurs at subsequent stages of visual processing (Allman et al., 1985a). In motion perception, center-surround interactions are found at all major processing stages (Tadin and Lappin, 2005a) and are particularly prominent

Received Oct. 5, 2005; revised Jan. 19, 2006; accepted Jan. 20, 2006.

This work was supported by National Institutes of Health Grant EY07760.

Correspondence should be addressed to Dr. Duje Tadin, Department of Psychology, 301 Wilson Hall, Vanderbilt University, Nashville, TN 37203. E-mail: duje.tadin@vanderbilt.edu.

DOI:10.1523/JNEUROSCI.4253-05.2006

Copyright $\odot 2006$ Society for Neuroscience $\quad$ 0270-6474/06/262614-09\$15.00/0 in MT (Allman et al., 1985b; Born and Tootell, 1992; Raiguel et al., 1995; Born, 2000), a cortical area vitally involved in motion perception (Born and Bradley, 2005). A typical center-surround neuron responds strongly when the preferred motion stimulus is imaged within the receptive field center of the neuron. But, if the spatial extent of stimulation is enlarged to include the surround, neural response attenuates: the neuron exhibits surround suppression. Such surround suppression, however, is typically restricted to high-contrast stimuli (Pack at al., 2005). At low contrast, the suppressive influences of the surround weaken, and spatial summation increases, a potentially adaptive mechanism that uses suppression only when the signal is sufficiently strong (Marr, 1982). These center-surround interactions enable neurons to perform complex operations, but likely require additional synaptic processing to incorporate surround signals (Raiguel et al., 1999). Indeed, the inhibitory component of the surround response in MT lags $\sim 10 \mathrm{~ms}$ after the center response (Borghuis et al., 2003).

Importantly, center-surround interactions have measurable perceptual correlates (Tadin et al., 2003; Betts et al., 2005; Paffen et al., 2006), but to dissect fine-scale temporal properties of surround suppression using psychophysical techniques presents a challenge. One potentially profitable strategy is to use stimulus manipulations that will differentially affect processing speed of center and surround signals. Such manipulations may affect the interaction between the center and surround, especially if the signals themselves are very brief. To implement this strategy, we 


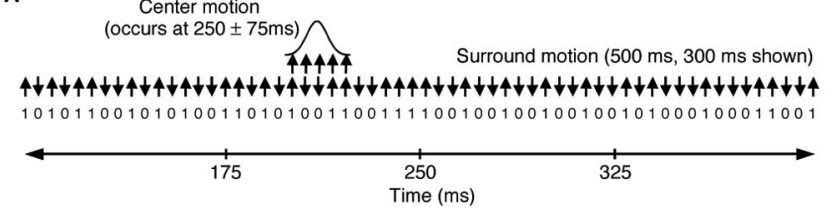

B

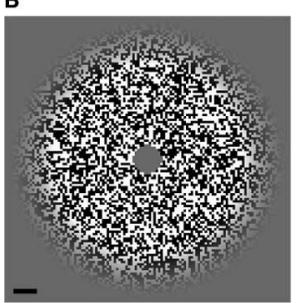

C

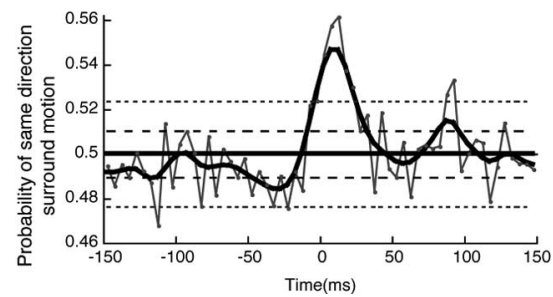

Figure 1. A, lllustration of the sequence of events occurring in the center and surround regions of the stimulus. Each arrow depicts a $5 \mathrm{~ms}$ motion impulse. All upward surround motions (i.e., those in the same direction as the center motion) are coded as 1, whereas all downward motions are coded as 0 . The Gaussian envelope over the center motion depicts contrast modulation of the center motion in time. $\boldsymbol{B}$, Single-frame snapshot of the surround pattern. The random-dot texture moved either up or down, with the direction chosen randomly every $5 \mathrm{~ms}$. Such motion sequences were typically perceived as very fast up- down jitter. The edge of the central hole, the location where the center motion appeared, was always stationary. Scale bar, $1^{\circ}$. C, Illustration of the raw data and the Gaussian smoothing of the data. The data sample is for the incorrect trials in the high-surround condition (92\%). The short dashed lines indicate \pm 2 SD boundaries for the raw data, whereas the long dashed lines indicate \pm 2 SD boundaries for the smoothed result.

designed a reverse correlation study in which the stimulus consisted of a brief $(\sim 20 \mathrm{~ms})$ center motion and a surround, the motion direction of which was randomly chosen every $5 \mathrm{~ms}$ (see Fig. $1 A, B)$. We then investigated whether the discriminability of center motion is influenced by surround motions occurring at different times relative to the center stimulus. Of particular interest was whether the timing of the influence of the surround changes with stimulus contrast, a question motivated by the known contrast-dependency of neuronal latencies (Lennie, 1981; Maunsell and Gibson, 1992; Maunsell et al., 1999; Reich et al., 2001).

\section{Materials and Methods}

Stimuli. Stimulus patterns were created in MATLAB with the Psychophysics Toolbox (Brainard, 1997) and Video Toolbox (Pelli, 1997) and were shown on a linearized monitor $(800 \times 600$ resolution, $200 \mathrm{~Hz})$. To achieve a high video refresh rate, which was important for the present experiment, we used a high-speed PROCALIX monitor (Totoku, Irving, TX) driven by a MP 960 graphics card (VillageTronic, Berlin, Germany); this combination provided a $200 \mathrm{~Hz}$ frame rate. Viewing was binocular at $83 \mathrm{~cm}$ (yielding $2 \times 2$ arcmin per pixel). Ambient illumination was 3.4 $\mathrm{cd} / \mathrm{m}^{2}$. Background luminance was $42.3 \mathrm{~cd} / \mathrm{m}^{2}$. Stimuli were moving random-dot textures. The size of each dot was $6 \times 6$ arcmin; a given dot could be dark or light, with equal probability. Three observers participated in the experiment (D.T. and two naive individuals). All procedures complied with institutionally reviewed guidelines for human subjects.

A moving random-dot surround (Fig. $1 B$ ) was presented in a spatial envelope consisting of a uniform annulus encircled by a blurred border (the spatial gradient of the border was a half cycle of the raised cosine function). The width of the uniform annulus section was $3.55^{\circ}$, and the width of the blurred border was $2.05^{\circ}$, resulting in the $12.3^{\circ}$ stimulus diameter, which is sufficiently large to stimulate the surrounds of neurons with foveal receptive fields in both V1 and MT (Raiguel et al., 1995; Jones et al., 2001). In the center of the moving random-dot texture was a small hole (diameter, $1.3^{\circ}$ ) in which the center random-dot pattern was briefly presented on each trial. The size of this center region is larger than the macaque V1 foveal receptive fields (Jones et al., 2001) but smaller than the receptive field centers in MT fovea (Raiguel et al., 1995). Moreover, the relatively small size of this center region ensured that the center stimulus on its own evokes little or no surround suppression (Tadin and Lappin, 2005b). The center contrast was ramped on and off with a temporal Gaussian envelope, the peak contrast of which was $92 \%$. In four different conditions, the surround contrasts were $92,27,6.5$, and $1.7 \%$.

The temporal sequence of events is illustrated in Figure $1 \mathrm{~A}$. The surround pattern was presented for $500 \mathrm{~ms}$, during which the dots in the surround texture moved uniformly upward or downward at $20 \%$ s (i.e., randomly jittered up-down). A new direction (up or down) was selected randomly every $5 \mathrm{~ms}$ (every frame on a $200 \mathrm{~Hz}$ monitor). At a randomly chosen time during the central $150 \mathrm{~ms}$, the center stimulus was briefly presented and moved either up or down at $20 \%$ s. The observers' task was to identify the direction of motion of the center pattern. The observers were instructed to fixate the center of the stationary gray circle at which center motion would occur and to maintain fixation throughout the 500 ms trial. In fact, consistently good performance required strict fixation, because the onset of the center motion was random. Performance was maintained near $70 \%$ correct by two interleaved adaptive staircases adjusting the center pattern duration [duration is defined as $1 \mathrm{SD}(\sigma)$ of the temporal Gaussian envelope controlling center contrast]. The temporal Gaussian envelope offers a temporal equivalent of subpixel sampling, permitting motion durations $(1 \sigma)<5 \mathrm{~ms}$. Duration thresholds in this experiment were very low (as low as $3 \mathrm{~ms}$ ) primarily because of the fast center speed. For example, a $3.91 \mathrm{~ms}$ threshold would be shown in five video frames with contrasts that are $3.8,44.1,100,44.1$, and $3.8 \%$ of the peak contrast (3.91 ms was the average threshold for $92 \%$ surround contrast condition) (compare Fig. $4 \mathrm{~A}$, leftmost point).

Analysis. To quantify the temporal aspects of center-surround interactions, we separately analyzed temporal sequences of surround motion that occurred during correct and during incorrect trials. For both types of trials, the specific goal was to look for patterns in the temporal sequence of surround motions that were different than expected by a random process. To perform such an analysis, the sequence of surround motions on each trial was coded relative to the center motion. Specifically, when any given $5 \mathrm{~ms}$ motion step in the surround was the same as the center motion, it was coded as 1 . When it was opposite to the center motion, it was coded as 0 . For every trial, this resulted in a temporal sequence of $0 \mathrm{~s}$ and $1 \mathrm{~s}$ with the origin (i.e., $0 \mathrm{~ms}$ point) aligned with the peak of the center motion. Correct and incorrect trial sequences were then averaged separately and smoothed with a moving Gaussian window. The SD $(\sigma)$ of the smoothing Gaussian was $7.5 \mathrm{~ms}$; this $\sigma$ removed most of the noise but did not affect the overall shape of the temporal profile. Figure $1 C$ shows an example of raw data and the result of smoothing. All of the analyses were done on smoothed data.

A central rationale behind this method is that if surround motion has no influence on the discriminability of the center motion, then after many trials, the result at each $5 \mathrm{~ms}$ time interval would approach 0.5 (i.e., equal proportion of the same and opposite directions in the surround). Departures from this value of 0.5 would indicate an influence of the surround on center motion discrimination. For example, if the occurrence of the same direction of motion at a specific $5 \mathrm{~ms}$ interval increases the likelihood of making a mistake, then the result for the incorrect trials at that time interval would be $>0.5$. Note that in a $2 \mathrm{AFC}$ (two alternative forced choice) design, the incorrect trials are more informative than the correct trials, because a correct response is often a correct guess. Hence, to increase the number of incorrect trials, a relatively high task difficulty was maintained (yielding 70\% correct performance).

The method yields meaningful results only after a large number of trials (i.e., when the random noise is "averaged out"). Thus, all observers ran 2500 trials per condition, with the first 500 trials discarded as practice. Observers typically ran 1000 trials during each experimental session lasting approximately $1 \mathrm{~h}$. The data shown in figures presented here required 66,400 trials. To quantify the variability expected by the random process alone, we ran 10,000 Monte Carlo simulations of the experiment assuming that surround motions have no influence on the discriminability of center motion. The results of each simulation were smoothed with a moving Gaussian window ( $\sigma=7.5 \mathrm{~ms}$ ), as shown in Figure 1C. This 
yielded 10,000 incorrect trial curves and 10,000 correct trial curves. Two SD boundaries were estimated from these two sets of curves.

This method is designed to yield a temporal profile of the surround influence on the center motion. This temporal profile was characterized by five parameters: (1) direction of influence (main peak/dip $>0.5$ or $<0.5$ ), (2) strength of influence (deviation of the peak influence from 0.5 ), (3) peak time (the time of the peak influence relative to the center motion), (4) start time (the earliest time at which significant influence is observed), and (5) duration of influence (time between the start time and the stop time).

Of particular interest is the determination of whether any of these parameters change with variations in surround contrast, because contrast will likely affect the timing of surround signals. In fact, the present method is optimized to estimate the relative latencies of surrounds with different contrasts and is not very sensitive to the absolute delay between center and surround signals. That is because the zero $(0 \mathrm{~ms})$ point in our graphs denotes the physical occurrence of the center motion. In other words, all comparisons are between stimulus-defined points in time for center and surround. A "real" zero point would be shifted rightward by the amount equal to the center neural response latency at a point in motion processing at which center and surround signals interact, a number unknown to us. Given that the brief center stimulus appears gradually within a temporal Gaussian envelope and attains its peak contrast only in one frame, and that its occurrence is random within $150 \mathrm{~ms}$, we speculate that its neural latency may be rather long. Moreover, a relatively long center latency may explain surround influence peaks occurring slightly ahead of the occurrence of the center motion (Fig. 1C), even if actual surround influence is delayed (cf. Allman et al., 1985b; Borghuis et al., 2003).

Low-contrast center experiment. We attempted to repeat the reverse correlation experiment just described with low-contrast center motion, but the results did not differ from chance. A likely problem was that the duration threshold for the low-contrast center motion was typically one order of magnitude higher than with high-contrast center motion. This longer exposure duration is critical because the success of the reverse correlation experiment depends on having a very brief target motion. To get around this problem, we repeated the experiment at low contrast with the following changes. (1) Duration thresholds for the low-contrast center were lowered by replacing the Gaussian temporal envelope with a trapezoid-like temporal envelope. This new profile was created by truncating the top half of the Gaussian profile and adding 0.5 (i.e., $50 \%$ contrast) to the truncated profile. For example, an $8.8 \mathrm{~ms}$ threshold (average threshold for the data shown in Fig. 7) would be shown in 11 5 -ms frames with contrasts that are 52, 57, 73, 100, 100, 100, 100, 100, 73, 57 , and $52 \%$ of the peak center contrast. These, in other words, are the contrast values obtained by applying the described transformation to a temporal Gaussian with $\sigma=8.8 \mathrm{~ms}$. This was still a relatively long stimulus presentation, but it was much shorter than the threshold stimulus duration when the standard Gaussian envelope was used. (2) To reduce the number of different surround directions that may have an influence on the discriminability of the center motion during each trial, a new direction of surround motion was chosen randomly every $10 \mathrm{~ms}$ or, in a separate condition, every $15 \mathrm{~ms}$. This maneuver meant that a given direction of motion in the surround continued for a longer duration, thereby reducing the number of motion direction changes in a given time period. (3) Center contrast was chosen to be relatively low but still high enough to yield moderately low thresholds. Based on the results of pilot explorations in which the contrast threshold was measured for a $10 \mathrm{~ms}$ presentation of the center motion, a $5.25 \%$ center contrast was used in the $10 \mathrm{~ms}$ surround motion condition, and a $6 \%$ contrast was used in the 15 ms surround condition.

\section{Results}

The discriminability of the center motion was substantially affected by the rapidly jittering surround pattern (Fig. 2). For all observers, surround temporal profiles estimated from incorrect trials significantly differed from what was expected by chance alone. Moreover, all temporal profiles are positioned primarily

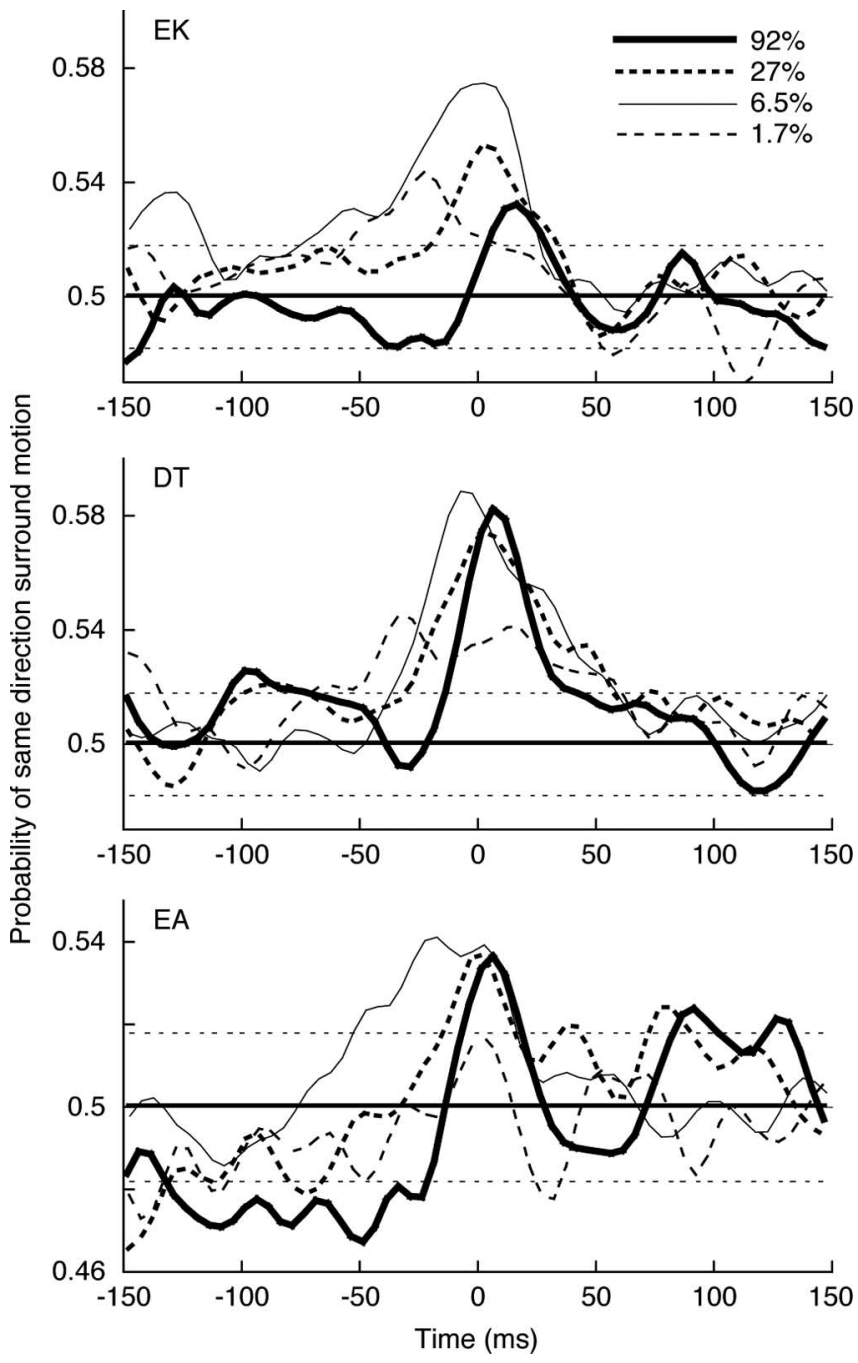

Figure 2. Temporal reverse correlation functions depicting incorrect trial results for three observers. Each curve represents data for a different surround contrast. Data were smoothed as illustrated in Figure 1C. The dashed horizontal lines are \pm 2 SDs.

above 0.5 , indicating that the presence of the same direction of motion in the surround increased the probability of making a mistake about the direction of the center motion. To derive an overall estimate of the temporal characteristics of the surround influence, the individual results were averaged (Fig. 3A). Quantitative estimates of these temporal characteristics are presented in Table 1. Peak time, start time (Fig. 3A, arrows), and duration of influence all showed a systematic dependence on surround contrast: the temporal profile of surround influence was longer, started earlier, and peaked earlier as the surround contrast was decreased. These results may be a result of longer and more variable neural response latencies at low contrast (Lennie, 1981; Maunsell et al., 1999; Reich et al., 2001).

The strength of the effect of the surround on the discriminability of center motion depended on the contrast of the surround (Table 1), with the $6.5 \%$ contrast surround having the strongest and "longest" effect on the center motion. If our estimates of the duration and the strength of influence indeed indicate the potency of the surround effect and if the effect of the surround is suppressive, then the center motion would be harder to perceive in conditions with stronger and longer surround influence. To test this hypothesis, we compared the potency of 

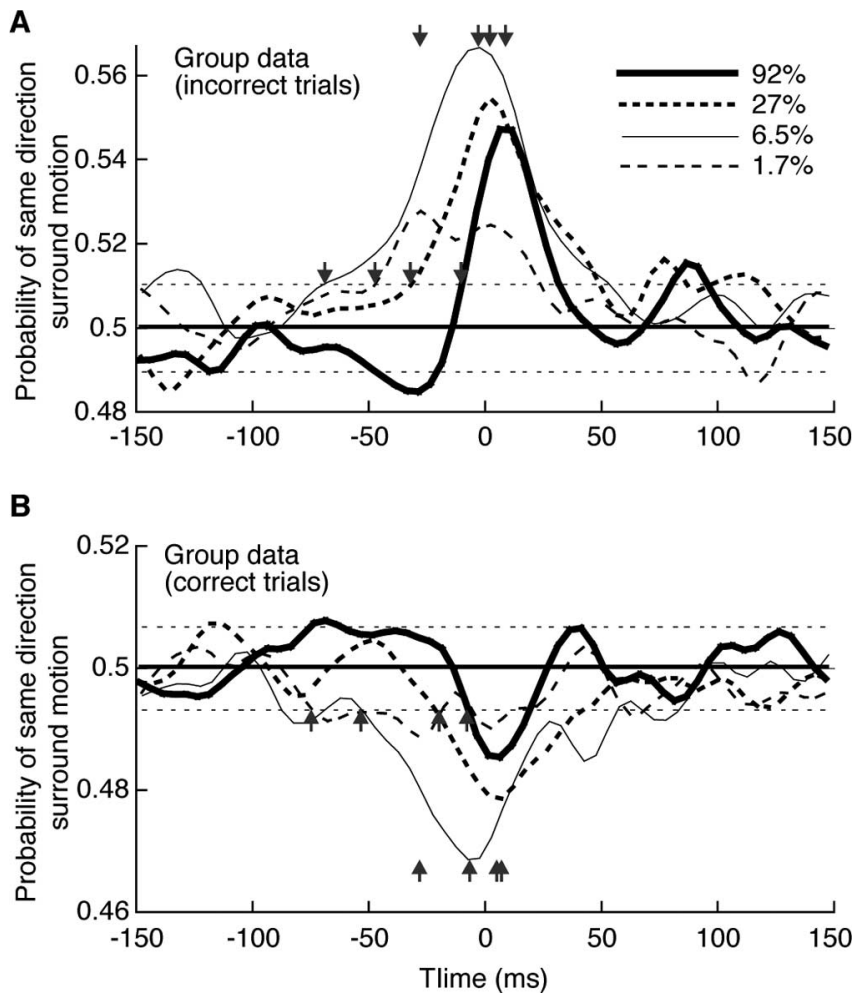

Figure 3. A, Temporal reverse correlation functions depicting incorrect trial results averaged over three observers. For each surround contrast, the arrows indicate the peak surround influence (top arrows) and the times at which surround influence reaches significance (arrows along the dashed line). $\boldsymbol{B}$, Temporal reverse correlation functions depicting correct trial results averaged over three observers. For each surround contrast, the arrows indicate the time point with the strongest surround influence (bottom arrows) and the times at which surround influence reaches significance (arrows along the dashed line). The dashed horizontal lines in both panels are \pm 2 SD boundaries.

surround influence in different conditions with the observers' thresholds in those conditions. For the quantitative part of this analysis, we excluded the data for the $1.7 \%$ surround contrast condition (Fig. 4, gray circles), because this very low contrast surround pattern perceptually partially filled-in the region where the center would appear, likely affecting the observers' performance. Analysis of the remaining three surround conditions (Fig. $4 A$ ) showed a positive correlation between duration thresholds and the duration of influence $(p=0.038)$, and there was a trend toward positive correlation with the strength of influence $(p=$ 0.13) (Fig. 4E). Positive correlations between duration thresholds and the estimates of surround influence were also observed when the data for individual observers were analyzed (Fig. $4 B-$ $D, F-H)$. This shows that the task was harder in the conditions with stronger and longer surround influence on the center mo- tion, as indexed by both the strength and the duration of influence.

For incorrect trials (Fig. 3A), the stronger surround influence indicates the occurrence of a larger number of same-direction surround motions and a smaller number of opposite-direction motions. Thus, the increasing task difficulty with the increasing surround influence could be caused either by an increase in samedirection suppression or by a reduction in opposite-direction facilitation. In other words, the results in Figure 4, although entirely consistent with surround suppression, do not rule out facilitation from opposite-direction surround motions. One way to distinguish between these two possible accounts is to compare psychophysical thresholds with the surround and with thresholds measured without the surround. If the effect of the surround is mostly facilitatory, then the addition of the surround should lower the thresholds. In contrast, if the surround is suppressive, the thresholds should increase. The average no-surround threshold was $3.2 \pm 0.4 \mathrm{~ms}$, which is lower than the thresholds in all of the surround conditions (Fig. 4). It should be noted that the no-surround thresholds were measured before the experiment, therefore practice effects, if any, would have benefited the surround conditions. Moreover, the surround is not necessarily always suppressive: these identical surrounds actually lower the threshold for discriminating low-contrast center motion (see below). Thus, when the results in Figure 4 are considered along with duration thresholds for discriminating center motion without the surround, we conclude that the center-surround interactions recovered through reverse correlation are suppressive. Such an interpretation agrees with the psychophysical work revealing strong suppressive interactions in motion perception (Tadin et al., 2003) and a lack of significant opposite-direction facilitation in macaque MT (Raiguel et al., 1995).

Significant deviations from randomness were also found when correct trials were analyzed, although our forced-choice procedure necessarily means that the "correct" trials also include trials on which the observer guessed correctly. Nonetheless, on correct trials, the temporal profiles are now $<0.5$ (Fig. $3 B$ ), indicating that observers were less likely to correctly identify motion direction when there was same-direction motion in the surround. This is in accord with the results extracted from incorrect trials. The relative properties of the temporal profiles for different contrast surrounds (e.g., relative ordering of peak time, start time, and the duration of the influence) are comparable to those estimated from the incorrect trials.

The stimulus used in these experiments was a broadband random-dot texture, the elements of which moved one pixel per frame. At high spatial frequencies, such a stimulus will always have motion components in both up and down directions. Moreover, this aliasing at high spatial frequencies will be more pronounced at high contrast because of poor contrast sensitivity to

Table 1. Quantitative description of the temporal profiles shown in Figure $3 \mathrm{~A}$

\begin{tabular}{|c|c|c|c|c|}
\hline & \multicolumn{4}{|l|}{ Surround contrast } \\
\hline & $92 \%$ & $27 \%$ & $6.5 \%$ & $1.7 \%$ \\
\hline Direction of influence & Same direction suppression & Same direction suppression & Same direction suppression & Same direction suppression \\
\hline Strength of influence & $0.547(9.4 \sigma)$ & $0.554(10.4 \sigma)$ & $0.567(12.9 \sigma)$ & $0.528(5.4 \sigma)$ \\
\hline Peak time (ms) & $8.2(0)$ & $3.1(-5)$ & $-2.0(-10)$ & $-27.0(-35)$ \\
\hline Start time (ms) & $-9.1(0)$ & $-30.8(-22)$ & $-69.6(-62)$ & $-46.9(-38)$ \\
\hline Duration of influence (ms) & 41 & 80 & 123 & 71 \\
\hline
\end{tabular}

The measures presented are defined in Materials and Methods. The strength of influence is also expressed in terms of SDs away from 0.5 (in parentheses). The peak time and start time are also shown as relative to $92 \%$ surround contrast estimates (in parentheses). To obtain a more precise estimate of peak and start times, we calculated a weighted average of data within $\pm 10 \mathrm{~ms}$ of the temporal point at which the surround influence was strongest, or, for the start point calculation, the point at which the significant influence was first observed. 

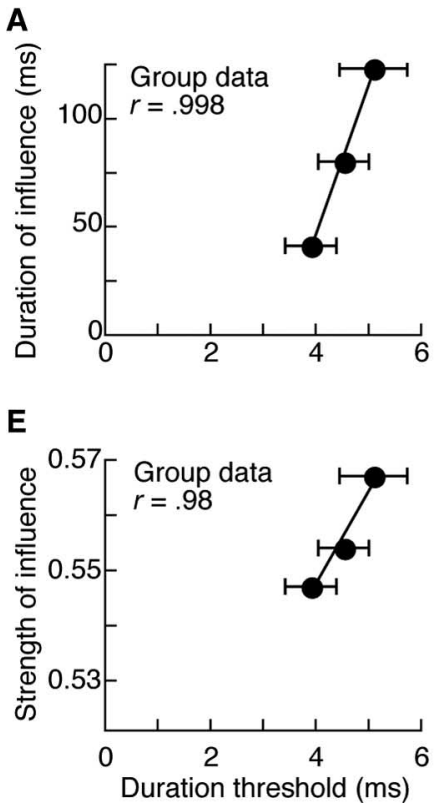

B

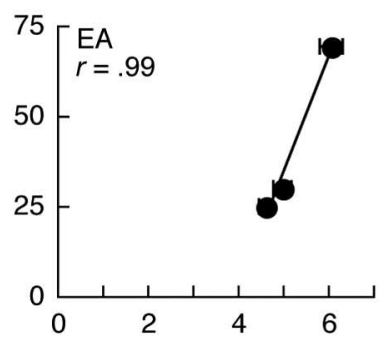

$\mathbf{F}$

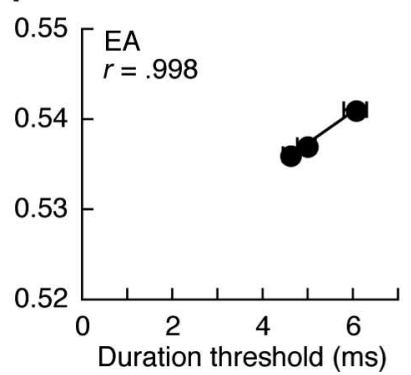

C

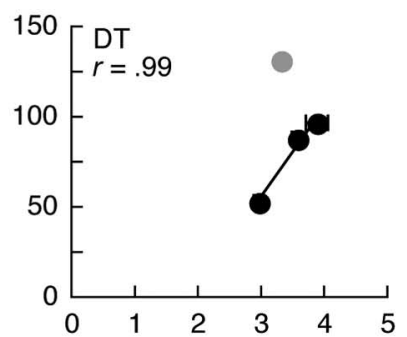

G

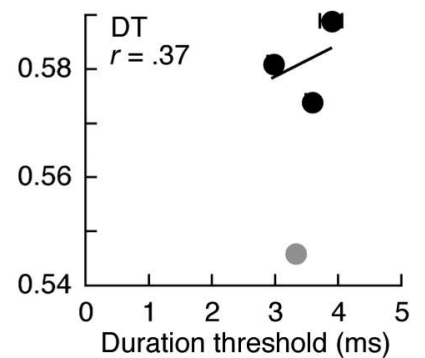

D

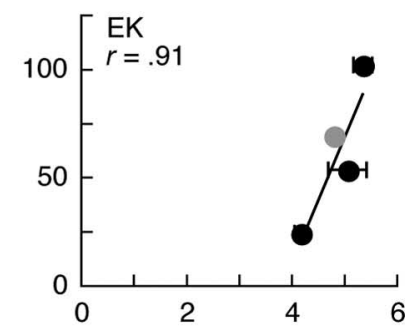

H

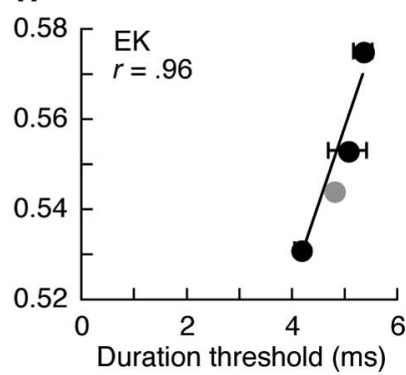

Figure 4. The relationship between psychophysical thresholds and two estimates of the potency of center-surround interactions: the duration of the surround influence (A-D) and the strength of the surround influence $(\boldsymbol{E}-\boldsymbol{H})$. $\boldsymbol{A}$ and $\boldsymbol{E}$ show the average data, whereas the remaining panels show results for individual observers. The straight lines are linear fits to the data. The gray circles depict results for the $1.7 \%$ surround contrast condition. No such data are shown for observer E.A., because his results for the $1.7 \%$ surround contrast condition did not differ from chance (see Fig. 2). His duration threshold in that condition was $6.7 \mathrm{~ms}$. Error bars indicate SEM.

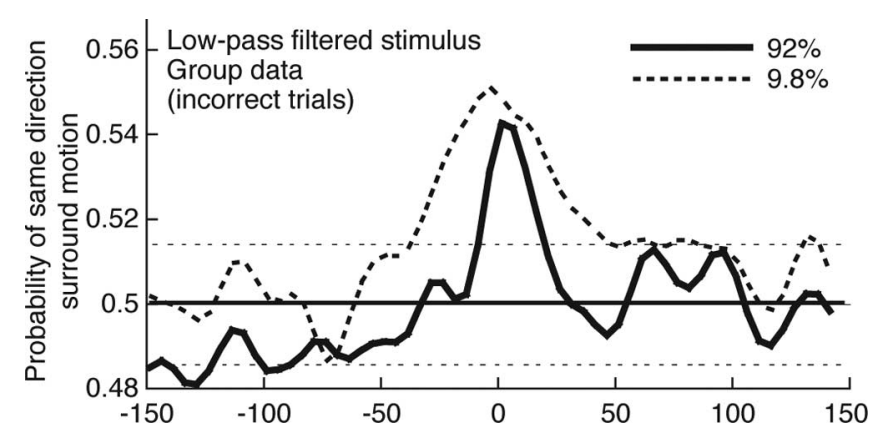

Figure 5. Temporal reverse correlation functions measured with low-pass-filtered stimuli. The data show results derived from incorrect trials obtained from two observers. The results were smoothed as illustrated in Figure 1 C. The dashed horizontal lines are \pm 2 SDs.

high spatial frequencies (van Nes and Bouman, 1967). For the present study, it is important to establish that this contrastdependent aliasing is not causing the differences among different surround contrast conditions. Thus, in a control experiment, we low-pass filtered our stimulus (cutoff frequency, 1.46 cycles/degree), effectively eliminating spatial frequency components susceptible to aliasing. Two surround contrasts were used ( 92 and $9.7 \%$ ) along with a high-contrast center $(92 \%)$. Note that because of spatial filtering, the stimulus consisted of a full range of contrasts and not just the extremes. This resulted in lower perceived contrast, requiring us to use a somewhat higher value for the "low" contrast condition. Still, the results (Fig. 5) were qualitatively similar to those obtained with broadband textures (stronger and longer effects at low contrast), indicating that contrastdependent aliasing cannot explain the pattern of results in Figure 3.

One interesting feature of our results is the "dip" appearing $\sim 35 \mathrm{~ms}$ before the peak in the $92 \%$ surround contrast condition (Fig. $3 A$ ). This dip is significant ( $>3 \sigma$ below baseline) and indicates that at those times the presence of the opposite-direction surround increased the probability of making a mistake. How- ever, $20-50 \mathrm{~ms}$ later, it is the same-direction surround motion that increases the likelihood of making an incorrect response. This temporal profile is reminiscent of the response of biphasic MT neurons (Bair and Movshon, 2004; Perge et al., 2005), which are best activated by an antipreferred direction, followed by a preferred direction $40 \mathrm{~ms}$ later.

To further investigate this feature of our results, we performed a second-order analysis of the data. To do this, we asked whether, within the data comprising each surround temporal profile (e.g., 92\% surround contrast condition in Fig. $3 A$ showing data from incorrect trials), different combinations of surround motion directions occurred more often than expected. The probability of each of four possible motion direction pairs (same-same, opposite-opposite, same-opposite, and opposite-same) was computed and compared with the expected baseline probabilities, in which the expected probability is simply the product of independent probabilities for each motion direction in the pair that is being examined. Probabilities were calculated for all motion direction pairs (i.e., the analysis was not limited to sequential motion directions). The results for all surround contrasts (data not shown) did not differ from what was expected given baseline (first-order) probabilities of each surround direction in a pair. This failure to find second-order effects demonstrates the independence of surround motion impulses. For example, the probability of "opposite" surround motion at the bottom of the dip in Figure $3 A$ ( $92 \%$ contrast) is 0.5255 , whereas the probability of the "same" surround motion at the peak is 0.5627 (these numbers are from the raw data) (Fig. 1C). The probability that this pair of surround motion directions occurred is 0.2957 , a value only slightly higher than what is expected if independence is assumed (0.2880). A second-order analysis looking at the pairs of motion directions within correct trials also failed to show any systematic deviations from chance. Such null results, however, are hard to interpret because of the smaller number of usable trials (each direction combination occurs only in approximately one-fourth of the trials). Moreover, any second-order effects are likely 

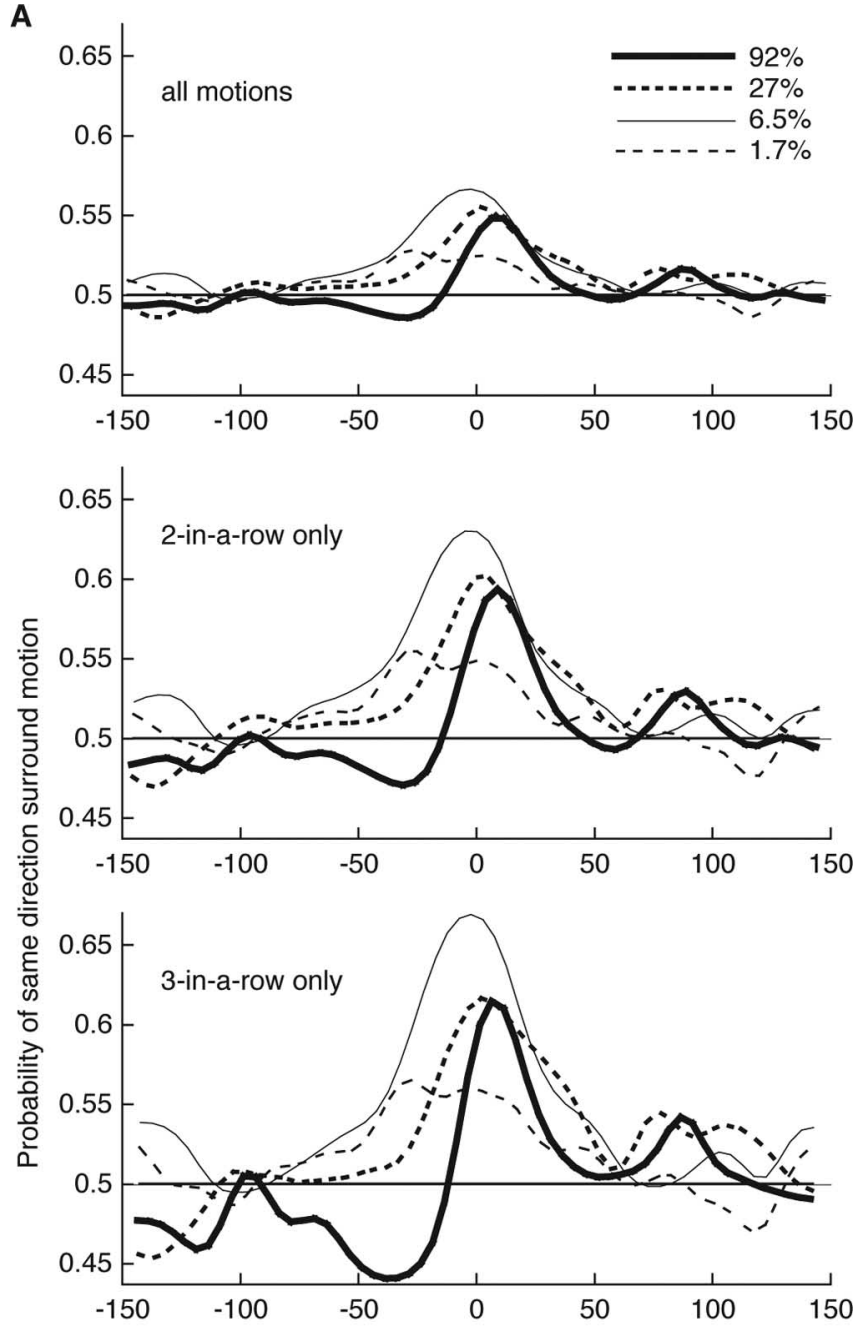

B

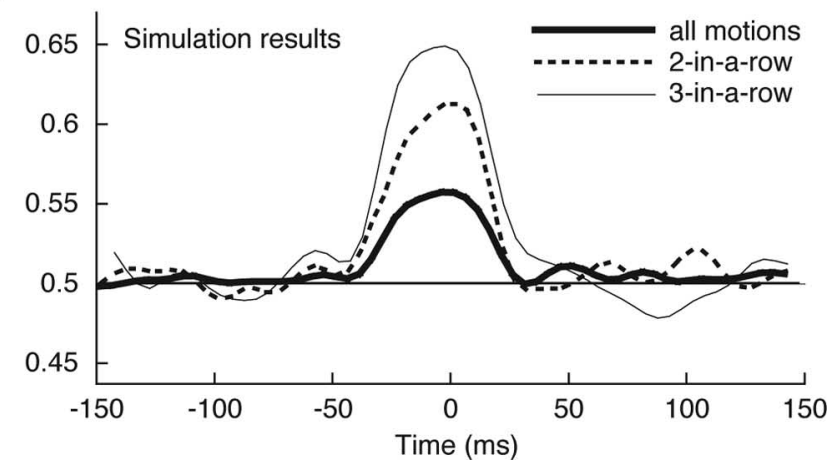

Figure 6. Results of the sequence-based analyses. $\boldsymbol{A}$, The sequence-based analysis of the psychophysical data. The data from Figure $3 A$ are replotted in the top panel. The bottom panels show the results from two-in-a-row and three-in-a-row analyses. Note that the $y$-axes are identical for all panels. It is clear that the strength of the surround grows when shorter sequences of surround motion impulses are excluded. However, the shape of the surround temporal profiles and, more importantly, their duration do not change very much. This observation suggests that the shorter duration of influence in conditions exhibiting weaker surround strength (e.g., 92\% contrast in Fig. 3A) are not attributable to an "iceberg effect" that could have caused weaker surround effects to appear substantially shorter in duration. $\boldsymbol{B}$, The sequence-based analysis of the simulation data. For the simulation, 6000 random surround motion series were generated, along with 6000 corresponding center motions (to equal the data collected from 3 observers). The surround motions were recorded as 1 (same) or 0 (different), depending on whether a surround motion was the same or different from the center motion. Then 10 surround motions in the middle of the series (central, $50 \mathrm{~ms}$ ) were designated as those potentially having an influence on the outcome of a trial. This effect was such that when no same-direction motions occurred, performance was $100 \%$. That is, when all 10 surround smaller in magnitude and thus harder to detect. In addition, second-order effects are compared with already noisy baseline probabilities, therefore further decreasing our chances of detecting them. Thus, we are uncertain whether the copresence of the dip and the peak in $92 \%$ contrast data indicates two independent effects or whether that result is attributable to an opposite-same interaction analogous to that observed in biphasic MT neurons (Pergé et al., 2005; Bair and Movshon, 2004).

In addition to pairwise second-order effects, we also examined whether sequential pairs and/or triplets of identical surround motions have a stronger effect on the discriminability of the center motion. To do this, we reanalyzed the data by taking into account only the identical pairs and triplets of surround motions. That is, for the "two-in-a-row" analysis, we considered only surround motion impulses that were in the same direction for two or more frames. Analogously, for the "three-in-a-row" analysis, we only included surround motion impulses that moved in the same direction for three or more frames. The results (Fig. 6A) show that the strength of the influence of the surround increased as shorter sequences of surround motion impulses are excluded. On average, two-in-a-row analysis resulted in 93\% stronger surround influence, whereas three-in-a-row analysis yielded a $235 \%$ increase in the strength of the influence of the surround (with "strength" referring to the deviation of the peak influence from 0.5 , as defined in Materials and Methods). This is perhaps not surprising because these analyses are based on biased sampling of surround motions. To assess the possible contribution of biased sampling, we ran a simple simulation in which each samedirection surround motion occurring within a $50 \mathrm{~ms}$ window had an independent inhibitory effect on the center motion (details in the figure legend). This resulted (Fig. 6B) in surround temporal profiles, the strength of which was similar to those measured psychophysically, although their shape was constrained by simulation parameters. Notably, simulation results exhibit sequencedependent increases in the surround strength similar to those measured with human observers (99\% for two-in-a-row analysis and $233 \%$ for three-in-a-row analysis). Thus, the results shown in Figure $6 \mathrm{~A}$ can be explained by the biased sampling of surround motions and suggest that the effects of brief ( $5 \mathrm{~ms}$ ) surround motion impulses are apparently independent.

The data presented in Figures 2-4 suggest that the influence of the surround is suppressive regardless of surround contrast. This is somewhat puzzling in light of other evidence pointing to surround facilitation when the entire stimulus is low contrast (Tadin et al., 2003; Pack at al., 2005). Note, however, that in our reverse correlation experiments, the contrast of the center region was always high. Perhaps, then, it is the contrast of the center but not the surround that determines the directionality of center-surround interactions. We tested this possibility by modifying our experimental design to enable the use of a low-contrast center stimulus (see Materials and Methods). The resulting reverse cor-

$\leftarrow$

motions were in the direction opposite from the center motion, the simulation always yielded a correct response. With all same-direction motions, performance was $40 \%$. The mixed cases followed a linear relationship between two extremes; thus, a case with five same- and five opposite-direction motions yielded average (i.e., threshold) performance (70\%). Note that two-in-a-row and three-in-a-row surround sequences were given no preferential treatment (i.e., the effects of surround motions were independent). After the outcome of all 6000 trials was determined, all of the incorrect trials were averaged and smoothed as illustrated in Figure 1C. Note that the purpose of this simulation is to examine the effect of the sequence-based analysis using surround motions that obey linear temporal summation and not to serve as a full model of the effect of the surround on the center motion. 

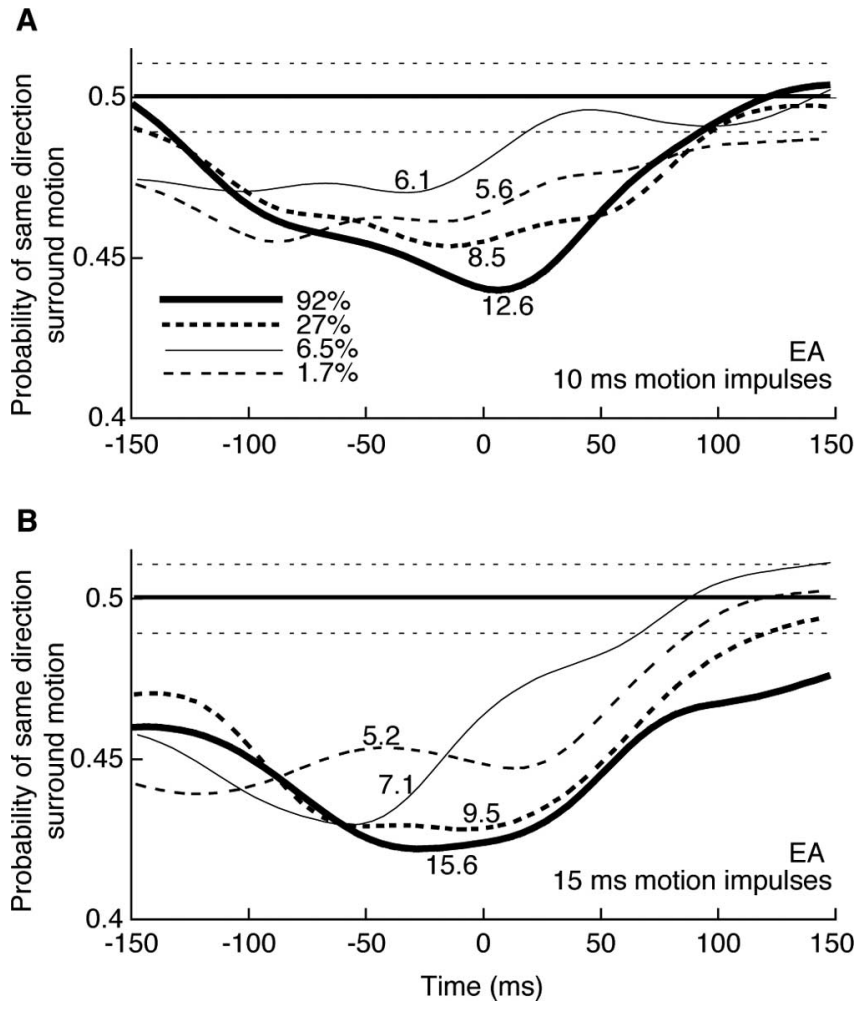

Figure 7. Temporal reverse correlation functions depicting incorrect trial results for observer E.A. in the low-contrast center condition, with surround motion directions constrained to move in one direction for $10 \mathrm{~ms}(\boldsymbol{A})$ and $15 \mathrm{~ms}(\boldsymbol{B})$. The numbers located near different curves indicate the psychophysical duration thresholds (in milliseconds) for each surround condition. The curves show smoothed raw data [smoothing was done with a moving Gaussian window ( $\sigma=$ $22.5 \mathrm{~ms}$ )]. Separate Monte Carlo simulations were performed to estimate 2 SD boundaries (shown as dashed lines). The details of the simulations were as described in Materials and Methods, except that surround motion parameters were adjusted to match the stimulus changes we made in this experiment.

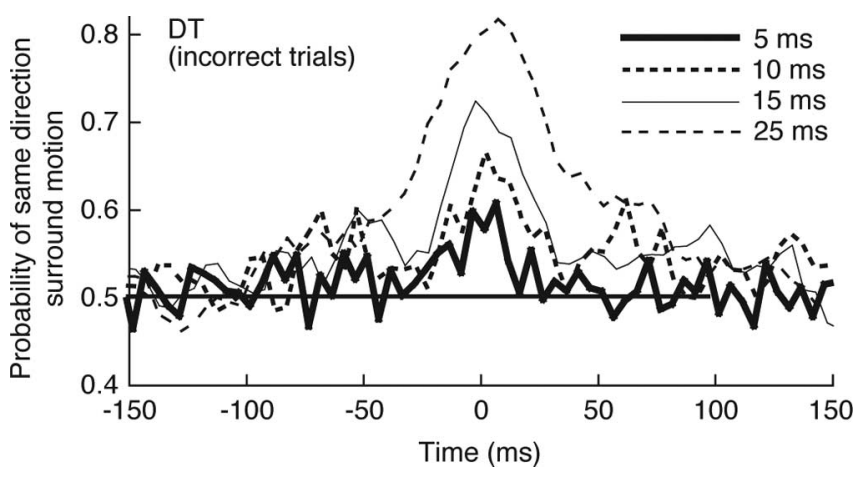

Figure 8. Temporal reverse correlation functions showing the results of a pilot experiment in which the duration of surround motion impulses was varied. The data show incorrect trials for one observer (D.T.). The experimental details are as described in Materials and Methods, except that each curve is based on 1600 trails.

relation temporal profiles were much broader than in the highcontrast center experiment (Fig. 7). This broadening is likely attributable to the longer center motion presentation and lengthening of surround motion impulses (to 10 and $15 \mathrm{~ms}$ ); our initial pilot work with high-contrast center motion with longer surround motions (10 and $15 \mathrm{~ms}$ ) also yielded broad surround temporal profiles (Fig. 8). Also, note that temporal profiles were broader when $15 \mathrm{~ms}$ surround impulses were used (Fig. 7B), again linking the changes in surround motion with observed broadening of the results.

The most important aspect of the low-contrast center result is that the temporal profiles derived from incorrect responses are inverted compared with profiles produced by the high-contrast center (compare Figs. 3A, 7). Moreover, by examining psychophysical thresholds in different conditions (shown as numbers near different curves in Fig. 7), it is apparent that the motion discrimination task was harder in conditions with stronger surround influence on the center motion. Given the directionality of the effect of the surround (i.e., the inversion), this indicates that there were fewer same-direction (and more opposite-direction) surround motions in incorrect trials. This result can indicate same-direction facilitation or opposite-direction suppression. To distinguish between these two possible accounts, we compared center duration thresholds with and without the surround. The thresholds for six of eight surround conditions were actually lower than the threshold for discriminating center motion presented without the surround (which was $10 \mathrm{~ms}$; see Materials and Methods). The only exceptions are duration thresholds for the $92 \%$ contrast surround, probably because the high-contrast surround texture reduced the effective center contrast (cf. Chubb et al., 1989). Generally, then, same-direction motion in the surround was associated with fewer mistakes, and the addition of the surround lowered direction discrimination thresholds. Evidently, the influence of the surround motion is now facilitative, whereas in the preceding experiments with high-contrast center, the influence was suppressive. In other words, the center contrast determines the directionality of center-surround interactions. Whether the interaction between the center and the surround is suppressive or facilitatory does not depend on surround contrast. The broad temporal influence functions for low-contrast center motion preclude accurate estimation of their temporal characteristics. Nevertheless, the peaks (i.e., dips) of the functions appear to have approximately the same dependence on surround contrast as the high-contrast center: progressively earlier peak times with decreasing contrast (Fig. 7).

\section{Discussion}

These experiments reveal interactions between center and surround motions and their dependency on relative direction, contrast, and timing. Same-direction surround motion reduced the discriminability of high-contrast center motion and improved the discriminability of low-contrast center motion, regardless of surround contrast. The timing of the observed center-surround interactions depended on surround contrast; as the contrast of the surround decreased, surround motion needed to occur earlier relative to the center motion to have an effect on the observers' performance.

These results were revealed using the temporal reverse correlation method, which allowed us to estimate the time course of center-surround interactions with relatively high precision, especially for very brief high-contrast center motion. It is worth reiterating that these results were obtained from surround motions that were perceived simply as a very fast up-down jitter. When queried, observers admitted that it was impossible to discern what surround motion sequence occurred just before and during center motion presentation; this aspect of the stimulus was invisible to perceptual awareness. Nonetheless, analysis of the raw data revealed significant and replicable patterns of surround influence on the discriminability of the center motion. In principle, similar results could have been obtained by presenting a brief surround motion pulse, followed by a brief center motion, 
at a range of temporal offsets (cf. Watson and Nachmias, 1977). The abrupt surround movement, however, might well engage transient, exogenous attention, which would affect the visibility of near-threshold center motion. Then any observed temporal interactions between the center and the surround motions would be confounded with the temporal effects of attention. On the other hand, the jittering surround stimulus we used was easily ignored (especially after a large number of practice trails), allowing our observers to easily focus attention to the center of the display.

Our results imply that neurons coding visual motion adequately represent rapidly changing motions that comprise surround stimuli used in the present study. Analyses of retinal responses to moving stimuli show that the direction of fast-moving stimuli can be encoded at a very fine time scale (Chichilnisky and Kalmar, 2003; Borghuis, 2003; Frechette et al., 2005). Optimal encoding of a $\sim 20 \%$ stimulus requires integration (i.e., low-pass filtering) of ganglion cell responses for $\sim 10 \mathrm{~ms}$, but sufficient information about motion direction is obtained even if the retinal responses are integrated for just over $1 \mathrm{~ms}$. This suggests that cortical motion mechanisms might be able to represent motion direction of very brief motion impulses. Cortical directionselective neurons indeed respond very well to stimuli randomly changing direction at $60 \mathrm{~Hz}$ (Buracas et al., 1998), $100 \mathrm{~Hz}$ (Bair at al., 2002; Bair and Movshon, 2004), or $120 \mathrm{~Hz}$ (Borghuis et al., 2003; Pergé et al., 2005). In fact, MT neurons respond with less variability and transfer information with higher efficiency when the stimulus rapidly changes direction (Buracas et al., 1998). In such cases, the spike timing precision to a repeated random sequence of directions can be as high as $2-3 \mathrm{~ms}$. Thus, although there are no studies of motion neurons with the $200 \mathrm{~Hz}$ change rate used here, direction-selective neurons respond with high fidelity to this general type of stimulation.

The longer neural latency associated with weaker visual stimuli (Maunsell et al., 1999; Reich et al., 2001) may explain differences in the start and peak time of the temporal profile for different contrast surrounds (Fig. 3A, Table 1). A "slow" low-contrast surround signal must be initiated well before the center signal to arrive in time to interact with the center signal. A "fast" highcontrast surround should be initiated only slightly before the center signal for the interaction to occur. Latencies of cortical neurons tend to increase by $30-40 \mathrm{~ms}$ as contrast decreases (Reich et al., 2001). Coincidentally or not, the peak of the surround temporal profile in our data shifted $\sim 35 \mathrm{~ms}$ back in time as the surround contrast decreased. In addition to the peak shift, we also observed broadening of temporal influence functions for low-contrast surrounds. This result may be explained by increased variability in the latency of low-contrast stimuli (Maunsell et al., 1999). Specifically, the increased range of surround latencies at low contrast extends the range of time points within which surround motion can occur and still interact with the center motion. In contrast, less variable latency of high-contrast stimuli restricts the range of relative temporal offsets for which the center and surround will interact.

We also found that the contrast of the center determines whether the spatial interactions in motion are suppressive or facilitatory (Fig. 7 vs Fig. 3A). This is an important observation that extends the initial psychophysical description of surround suppression (Tadin et al., 2003). Tadin et al. (2003) did not vary the contrasts of the center and surround separately, precluding any conclusions about whether the center or the surround contrast determines the sign of their interaction. The present finding is consistent with modeling work in which the activity level of the classical receptive field (i.e., center) determines the nature of center-surround interactions. Specifically, surround suppression is hypothesized to occur at high levels of center activity, whereas surround facilitation should be more pronounced at low levels of center activity (Stemmler et al., 1995; Somers at al., 1998). It should be noted, however, that models based on increased spatial summation at low contrast (cf. Sceniak et al., 1999) might also be consistent with the aforementioned results, although it is unclear how such models would respond to a low-contrast stimulus encircled by a high-contrast surround.

The center-driven transition from spatial suppression at high contrast to spatial summation at low contrast makes sense from a functional standpoint. At high contrast, sensitivity is not a primary concern, and it can be sacrificed in favor of processes that enhance spatial resolution and/or processes that extract useful information about differential motion defining object boundaries. On the other hand, sensitivity becomes increasingly more important as contrast decreases. Thus, it makes functional sense to integrate signals over space even if the cost is a loss in spatial resolution. For example, motion-based figure-ground segregation is impaired at medium and low contrasts, even in cases when motion sensitivity is relatively unaffected (Regan and Beverley, 1984; Regan, 1989; Takeuchi et al., 2004). This result is consistent with a system that shifts emphasis from spatial differentiation to spatial summation as the contrast decreases. What our results suggest is that the nature of these spatial interactions over space depends on the center contrast and not the surround contrast. As an aside, our results were obtained under conditions in which attention was undoubtedly focused on the center motion; our observers discriminated the motion of a very brief target that occurred at a random time during the stimulus presentation. Whether attention plays an important role in this adaptive contrast-dependency of center-surround interactions thus remains an open question.

As a final note, an important motivator for using the reverse correlation in neurophysiology is its time-efficiency-reverse correlation can be computed for every spike. In contrast, the psychophysical reverse correlation described here is extremely time inefficient. Instead of using frequent spikes as the basis of the computation, we rely on infrequent observers' responses. Nevertheless, the potential benefits of this technique can be well worth the extra effort. The psychophysical temporal reverse correlation may be adapted to investigate other visual mechanisms in which different components of the response are likely to have different temporal profiles such as iso-orientation suppression (Bair et al., 2003), figure-ground discrimination (Lamme, 1995), and illusory contour processing (Ramsden et al., 2001).

\section{References}

Allman J, Meizin F, McGuiness E (1985a) Stimulus specific responses from beyond the classical receptive field: neurophysical mechanisms for localglobal comparisons in visual neurons. Annu Rev Neurosci 8:407-430.

Allman J, Meizin F, McGuiness E (1985b) Direction- and velocity-specific responses from beyond the classical receptive field in the middle temporal visual area (MT). Perception 14:105-126.

Bair W, Movshon JA (2004) Adaptive temporal integration of motion in direction-selective neurons in macaque visual cortex. J Neurosci 24:7305-7323.

Bair W, Cavanaugh JR, Smith MA, Movshon JA (2002) The timing of response onset and offset in macaque visual neurons. J Neurosci 22:3189-3205.

Bair W, Cavanaugh JR, Movshon JA (2003) Time course and time-distance relationships for surround suppression in macaque V1 neurons. J Neurosci 23:7690-7701. 
Bedell HE, Chung STL, Ogmen H, Patel SS (2003) Color and motion: which is the tortoise and which is the hare? Vision Res 43:2403-2412.

Betts LR, Taylor CP, Sekuler AB, Bennett PJ (2005) Aging reduces centersurround antagonism in visual motion processing. Neuron 45:361-366.

Borghuis BG (2003) Spike timing precision in the visual front-end. Utrecht, The Netherlands: Utrecht University Library.

Borghuis BG, Perge JA, Vajda I, van Wezel RJA, van de Grind WA, Lankheet MJM (2003) The motion reverse correlation (MRC) method: a linear systems approach in the motion domain. J Neurosci Methods 123:153-166.

Born RT (2000) Center-surround interactions in the middle temporal visual area of the owl monkey. J Neurophysiol 84:2658-2669.

Born RT, Bradley DC (2005) Structure and function of visual area MT. Annu Rev Neurosci 28:157-189.

Born RT, Tootell RBH (1992) Segregation of global and local motion processing in primate middle temporal visual area. Nature 357:497-499.

Brainard DH (1997) The Psychophysics Toolbox. Spat Vis 10:433-436.

Buracas GT, Zador AM, DeWeese MR, Albright TD (1998) Efficient discrimination of temporal patterns by motion-sensitive neurons in primate visual cortex. Neuron 20:959-969.

Chichilnisky EJ, Kalmar RS (2003) Temporal resolution of ensemble visual motion signals in primate retina. J Neurosci 23:6681-6689.

Chubb C, Sperling G, Solomon JA (1989) Texture interactions determine perceived contrast. Proc Natl Acad Sci USA 86:9631-9635.

Frechette ES, Sher A, Grivich MI, Petrusca D, Litke AM, Chichilnisky EJ (2005) Fidelity of the ensemble code for visual motion in primate retina. J Neurophysiol 94:119-135.

Jones HE, Grieve KL, Wang W, Sillito AM (2001) Surround suppression in primate V1. J Neurophysiol 86:2011-2028.

Lamme VA (1995) The neurophysiology of figure-ground segregation in primary visual cortex. J Neurosci 15:1605-1615.

Lamme VA, Super H, Spekreijse H (1998) Feedforward, horizontal, and feedback processing in the visual cortex. Curr Opin Neurobiol 8:529-535.

Lennie P (1981) The physiological basis of variations in visual latency. Vision Res 21:815-824.

Marr D (1982) Vision. New York: Freeman

Maunsell JH, Gibson JR (1992) Visual response latencies in striate cortex of the macaque monkey. J Neurophysiol 68:1332-1344.

Maunsell JH, Ghose GM, Assad JA, McAdams CJ, Boudreau CE, Noerager BD (1999) Visual response latencies of magnocellular and parvocellular LGN neurons in macaque monkeys. Vis Neurosci 16:1-14.

Moutoussis K, Zeki S (1997) A direct demonstration of perceptual asynchrony in vision. Proc R Soc Lond B Biol Sci 264:393-399.

Nishida S, Johnston A (2002) Marker correspondence, not processing latency, determines temporal binding of visual attributes. Curr Biol 12:359-368.

Nowak LG, Bullier J (1997) The timing of information transfer in the visual system. In: Cerebral cortex, extrastriate cortex in primates, Vol 12 (Rocklund K, Kaas J, Peters A, eds), pp 205-241. New York: Plenum.

Pack CC, Hunter JN, Born RT (2005) Contrast dependence of suppressive influences in cortical area MT of alert macaque. J Neurophysiol 93:1809-1815.

Paffen CL, Tadin D, Te Pas SF, Blake R, Verstraten FA (2006) Adaptive center-surround interactions in human vision revealed during binocular rivalry. Vision Res 46:599-604.

Pelli DG (1997) The VideoToolbox software for visual psychophysics: transforming numbers into movies. Spat Vis 10:437-442.

Perge JA, Borghuis BG, Bours RJ, Lankheet MJ, van Wezel RJ (2005) Temporal dynamics of direction tuning in motion-sensitive macaque area MT. J Neurophysiol 93:2104-2116.

Raiguel SE, Van Hulle MM, Xiao DK, Marcar VL, Orban GA (1995) Shape and spatial distribution of receptive fields and antagonistic motion surround in the middle temporal area (V5) of the macaque. Eur J Neurosci 7:2064-2082.

Raiguel SE, Xiao DK, Marcar VL, Orban GA (1999) Response latency of macaque area MT/V5 neurons and its relationship to stimulus parameters. J Neurophysiol 82:1944-1956.

Ramsden BM, Hung CP, Roe AW (2001) Real and illusory contour processing in area V1 of the primate: a cortical balancing act. Cereb Cortex 11:648-665.

Regan DM (1989) Orientation discrimination for objects defined by relative motion and objects defined by luminance contrast. Vision Res 29:1389-1400.

Regan DM, Beverley KI (1984) Figure-ground segregation by motion contrast and by luminance contrast. J Opt Soc Am A 1:433-442.

Reich DS, Mechler F, Victor JD (2001) Temporal coding of contrast in primary visual cortex: when, what, and why. J Neurophysiol 85:1039-1050.

Sceniak MP, Ringach DL, Hawken MJ, Shapley R (1999) Contrast's effect on spatial summation by macaque V1 neurons. Nat Neurosci 2:733-739.

Somers DC, Todorov EV, Siapas AG, Toth LJ, Kim DS, Sur M (1998) A local circuit approach to understanding integration of long-range inputs in primary visual cortex. Cereb Cortex 8:204-217.

Stemmler M, Usher M, Niebur E (1995) Lateral interactions in primary visual cortex: a model bridging physiology and psychophysics. Science 269:1877-1880.

Tadin D, Lappin JS (2005a) Linking psychophysics and physiology of center-surround interactions in visual motion processing. In: Seeing spatial form (Jenkin MRM, Harris LR, eds), pp 279-314. Oxford: Oxford UP.

Tadin D, Lappin JS (2005b) Optimal size for perceiving motion decreases with contrast. Vision Res 45:2059-2064.

Tadin D, Lappin JS, Gilroy L, Blake R (2003) Perceptual consequences of centre-surround antagonism in visual motion processing. Nature 424:312-315.

Takeuchi T, Yokosawa K, De Valois KK (2004) Texture segregation by motion under low luminance levels. Vision Res 44:157-166.

Thorpe S, Fize D, Marlot C (1996) Speed of processing in the human visual system. Nature 381:520-522.

van Nes FL, Bouman MA (1967) Spatial modulation transfer in the human eye. J Opt Soc Am 57:401-406.

Watson AB, Nachmias J (1997) Patterns of temporal interaction in the detection of gratings. Vision Res 17:893-902. 